\title{
Antioxidant Levels in the Pig Urinary Bladder: Distribution within the Bladder Wall and in the Urothelium Derived from Different Bladder Regions
}

\author{
Tomaž Vovk,* Marija BogataJ, and Aleš Mrhar \\ Faculty of Pharmacy, University of Ljubljana; Aškerčeva 7, 1000 Ljubljana, Slovenia. \\ Received September 15, 2008; accepted February 3, 2009; published online February 12, 2009
}

This study was designed to determine the antioxidant levels in the urinary bladder wall layers as well as urothelium derived from different bladder regions. Samples of the urothelium, lamina propria, muscularis, and serosa were prepared from the pig's urinary bladder body, while samples used for regional mapping of the urothelium were prepared from trigone, ventral and dorsal middle bladder body, and apex region. Activities of superoxide dismutase, glutathione peroxidase, glutathione reductase, and catalase were determined. Concentrations of ascorbic acid and glutathione were also measured. Antioxidant activities, i.e. concentrations of superoxide dismutase, glutathione peroxidase, glutathione reductase and glutathione, were shown to be highest in the urothelium and progressively lower towards the serosa. Regional mapping of the urothelium singled out apex as the region with the lowest antioxidant activities, i.e. concentrations of glutathione peroxidase, ascorbic acid, and glutathione. The fact that antioxidants are concentrated in the urothelium implies that urothelium functions as a barrier against reactive species. The urothelium derived from the apex is the region with the lowest antioxidant levels and is therefore probably the region most liable to development of oxidative damage.

Key words urinary bladder; urothelium; antioxidant enzyme; low molecular weight antioxidant

There is increasing evidence indicating that oxidative stress is associated with various urinary bladder disorders. Oxygen and nitrogen reactive species formed during oxidative stress can produce oxidative cellular damage which can lead to urinary bladder dysfunction. To cope with reactive species, biological systems possess a range of defence mechanisms, the first line of which are antioxidants. Under physiological conditions, antioxidants are kept in balance with the production of reactive species to prevent cell damage. However, when production of reactive species is increased or the levels of antioxidants are diminished, the antioxidant defence is exceeded and oxidative damage of cellular macromolecules occurs.

Oxidative stress of the urinary bladder, as a consequence of increased production of reactive species, is mostly studied in relation to nitric oxide. Increased production of nitric oxide or expression of nitric oxide synthase has been demonstrated in bladder tumour cells, partial bladder obstruction, urinary tract infection, cystitis and ischemia/reperfusion. ${ }^{1-9)}$ On the other hand, decreased antioxidant enzymes levels, as well of low molecular weight antioxidants, have also been reported in urinary bladder cancer, urolithiasis, and sepsis. ${ }^{10-13)}$ The inevitable consequence of such conditions is oxidative damage. The latter was studied in bladder cancer, sepsis, ischemia/reperfusion, partial outlet bladder obstruction, aging, and low doses of ionizing radiation. Increases in malondialdehyde, ${ }^{11,13-16)}$ 3-nitrotyrosine ${ }^{17)}$ and 8-hydroxy$2^{\prime}$-deoxyguanosine ${ }^{18)}$ concentrations - biomarkers of lipid, protein, and DNA oxidation, respectively-have been found.

Since oxidative stress is present in both physiological and pathological conditions, organisms have had to adapt to it. Despite all the evidence confirming the important role of oxidative stress in the urinary bladder, no detailed research on the bladder antioxidants and their distribution within the bladder wall has been performed. The aim of the present study was to determine the urinary bladder distribution of an- tioxidant enzymes superoxide dismutase (SOD), catalase (CAT), glutathione peroxidase (GPx), and glutathione reductase (GR), as well as of major water soluble low molecular weight antioxidants, i.e. ascorbic acid (AA) and glutathione (GSH). Their concentrations were determined in urinary bladder wall layers and urothelium derived from various bladder regions. By mapping the urinary bladder we aimed to identify regions with the lowest antioxidant levels, and consequently highest sensitivity to oxidative damage and bladder disease. Such information may be crucial for understanding the initiation of pathological processes in urinary bladder.

\section{MATERIALS AND METHODS}

Bladder Tissue Porcine urinary bladders were obtained from the local slaughterhouse, where pigs ( 7 month-old male pigs, weighing $90-110 \mathrm{~kg}$ ) were bled to death after narcotization by electric shock, according to European Community regulations. After opening the abdominal wall, the bladder was excised, carefully emptied, rinsed with ice cooled and carbogen $\left(95 \% \mathrm{O}_{2}, 5 \% \mathrm{CO}_{2}\right)$ saturated phosphate buffered saline $\mathrm{pH} \mathrm{7.4,} \mathrm{and} \mathrm{transferred} \mathrm{into} \mathrm{the} \mathrm{buffer} \mathrm{of} \mathrm{the} \mathrm{same}$ composition for further treatment.

Tissue pieces of urinary bladder were cut from trigone, apex and middle parts of the ventral and dorsal bladder body as follows (Fig. 1A). The isolated bladder was immediately opened by a transverse incision on the ventral side between bladder body and base, followed by a longitudinal incision in the direction towards the urethra. Half way between the bladder apex and ureters the middle parts of the ventral and dorsal bladder body region were excised, and finally the apex region was isolated (Fig. 1B). Tissue pieces (mucosal and serosal surface of approximately $8 \mathrm{~mm}$ diameter) were cut and used for two different experiments of antioxidants determination. In the first experiment five tissue pieces were cut from the middle dorsal bladder body region. Each tissue 


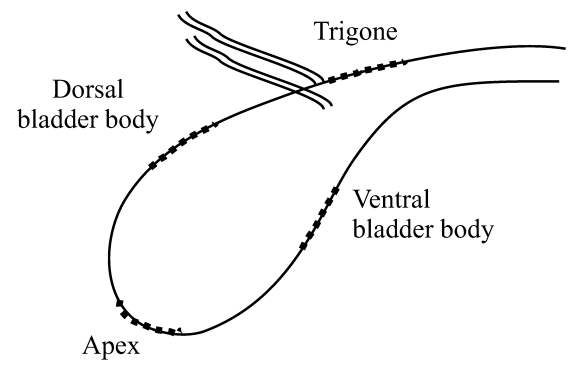

A

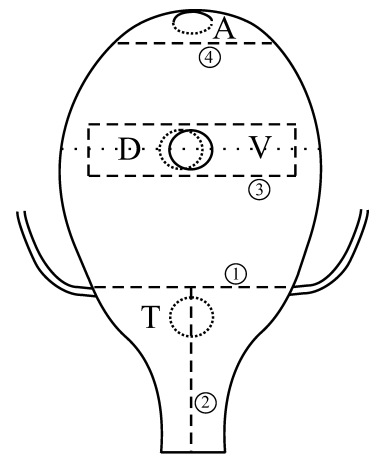

B

Fig. 1. (A) Urinary Bladder with Marked Regions Used for Tissue Sampling

The figure illustrates the bladder position and shape in quadrupeds, as seen from the lateral direction.

(B) Ventral View of the Isolated Urinary Bladder with Marked Regions for Tissue Sampling

The regions where bladder was incised and the order of incisions are marked with dashed line and numbers. The dotted line indicates the half way point between the bladder apex and ureters. T, Trigone; V, Middle Ventral Bladder Body; D, Middle Dorsal Bladder Body and A, Apex (Solid and dashed circles indicate ventral and dorsal region, respectively).

piece was cut through the muscular layer into two parts to obtain one part with the mucosal (mucosal piece) and the other with the serosal layer (serosal piece). Tissue pieces were blotted dry, placed with mucosal and serosal sides on two flat, parallel stainless plates with adjustable distance and frozen rapidly in liquid nitrogen. In the second experiment only one tissue piece was cut from each region and frozen rapidly in liquid nitrogen by the same procedure as described above. The frozen tissue pieces were kept at $-70^{\circ} \mathrm{C}$ for a maximum of two weeks before analysis.

Cryotome Sectioning of the Bladder Tissue The cryotome sectioning technique was used for preparing tissue samples.

In the preliminary experiments, the thickness of urinary bladder wall layers was estimated using frozen tissue pieces derived from the middle dorsal body. The tissue pieces were sectioned perpendicularly to the mucosal surface using a cryotome (Leica CM 1850, Germany) and haematoxylin-eosin stained images of sections were acquired using microscope (Olympus BX 50, Japan) and video camera (Sony 3CCD color DXC-950P, Japan). The thickness of layers was measured with the image-analysing software AnalySIS. The appropriate interval for tissue pieces sectioning was chosen, on the basis of estimated thickness, to produce tissue samples containing predominantly only one bladder layer. Sections from mucosal tissue pieces, starting at the mucosal side, from 0 to $100 \mu \mathrm{m}, 100$ to $200 \mu \mathrm{m}$ and 2000 to $2100 \mu \mathrm{m}$ in depth, represented urothelium, lamina propria, and muscularis samples, respectively. The serosal tissue sample was obtained by sectioning serosal tissue pieces, starting at the serosal side from 0 to $200 \mu \mathrm{m}$ in depth.

For the first experiment the mucosal and serosal tissue pieces were sectioned into samples containing urothelium, lamina propria, muscularis or serosa. Prior to sectioning the depth of urothelium layer was controlled in one of the five mucosal tissue pieces belonging to the same urinary bladder, using the same method as described above except for the staining. The section image was obtained by dark-field microscopy. If the urothelial layer was less than $20 \mu \mathrm{m}$ thick, all tissue pieces from the corresponding bladder were excluded from the study. Otherwise, tissue pieces were sectioned parallel to the mucosal or serosal surface and the specific bladder layer was obtained by sectioning the tissue pieces using the same procedure as described in preliminary experiment. The urothelial or serosal surface position was precisely adjusted to align its surface with the cryotome blade. Twentymicrometer thick sections were placed into pre-weighed plastic microcentrifuge tubes. The tissue sample of a specific bladder layer was obtained by combining sections of the selected layer from all five tissue pieces of the urinary bladder. The tissue samples were immediately used for antioxidant determination. The number of animals used for the first experiment is five and is equal to the number of samples.

For the second experiment the urothelium samples were prepared from tissue pieces derived from different bladders regions. First, the depth of the urothelium layer in each tissue piece was determined as described above. The number of $20 \mu \mathrm{m}$ thick sections was determined according to the urothelium depth. These sections were sectioned parallel to the mucosal surface from the inspected tissue piece. As the concentration of antioxidants in a urothelium sample from a specific region of one urinary bladder was too low to be determined, urothelium sections derived from the specific region of four to six urinary bladders were combined and constituted one urothelium sample used for analysis. The antioxidant analysis was performed on five different samples prepared from 20 to 25 animals.

Antioxidant Measurements-Antioxidant Enzymes Fifty millimolar phosphate buffer $\mathrm{pH} 7.0$ containing $0.1 \%$ Triton X-100 was added to the weighed tissue samples to obtain a concentration of $0.05 \mathrm{mg}$ of wet tissue per $\mu \mathrm{l}$ of extraction solution. After $1 \mathrm{~min}$ of homogenisation the homogenate was centrifuged for $15 \mathrm{~min}$ at $5^{\circ} \mathrm{C}$ and $20000 \mathrm{rpm}$. The supernatant was used for enzyme analysis.

SOD activity was measured using the xanthine-oxidase, xanthine and water soluble tetrazolium salt. ${ }^{19)}$ One unit of SOD activity is defined as the amount of sample producing $50 \%$ inhibition of the rate of water soluble tetrazolium salt reduction in the assay. The activity is expressed as units/g of wet tissue weight. GPx activity was assayed with a coupled 
enzyme system in which oxidized glutathione (GSSG) reduction was coupled to nicotinamide adenine dinucleotide phosphate (NADPH) oxidation by glutathione reductase. ${ }^{20)}$ Cumene hydroperoxide was used as a substrate for GPx. GPx activity was expressed in units/g of wet tissue weight and 1 unit presents $1 \mu \mathrm{mol}$ of NADPH oxidized/min. GR assay was based on reduction of 5,5'-dithiobis(2-nitrobenzoic acid) (DTNB) by GSH generated from GSSG and NADPH. It was measured according to the method described by Cribb et $a l .{ }^{21)}$ with slight modifications. ${ }^{22)}$ GR activity was expressed in units/g of wet tissue weight, assuming that 1 unit GR reduces $1 \mu \mathrm{mol}$ of DTNB/minute. CAT activity was assayed by monitoring decomposition of hydrogen peroxide and the enzyme activity is expressed as the rate constant of hydrogen peroxide decomposition. ${ }^{23)}$

Antioxidant Measurements-Low Molecular Weight Antioxidants (LMWA) LMWA were extracted from tissue samples using the same method as described in the antioxidant enzyme section except that $5 \%$ meta-phosphoric acid with $2 \mathrm{~mm}$ ethylenediamine tetraacetic acid was used as the extraction solution. AA and GSH were determined using HPLC method with electrochemical detector published elsewhere. $^{24)}$

Statistical Analysis The results are presented as means \pm S.D. Statistical analyses were performed with SPSS version 12.0 for Windows (Statistical Package for the Social Science, SPSS Inc., Chicago, IL, U.S.A.). The statistical significance of results was assessed using the univariant ANOVA followed by post hoc Bonferroni's test. Significance was considered at $p<0.05$.

\section{RESULTS}

The cryotome sectioning technique enabled us to obtain tissue samples derived predominantly from specific bladder wall layers. The histology figures of the pig urinary bladder wall obtained after the haematoxylin-eosin staining procedure and dark-field microscopy are presented in Fig. 2. The average thicknesses of the urothelium, lamina propria, muscularis, and serosa derived from the middle dorsal bladder body were $68 \pm 17,657 \pm 148,5550 \pm 1168$, and $90 \pm 26 \mu \mathrm{m}$. The mean thickness of the urothelium layer derived from different bladder regions ranged from 65 to $82 \mu \mathrm{m}$. The urothelium thickness did not differ between different regions.

The antioxidant distribution in the urinary bladder wall studied on the middle dorsal bladder body region is presented in Fig. 3. The activities were studied on five samples obtained from five pigs. The activities of all antioxidant enzymes except CAT were highest in the urothelium, and progressively lower from urothelium to serosa. The activities of SOD, GPx, GR, and CAT in the urothelium sample were $423 \pm 83 \mathrm{U} / \mathrm{g}, \quad 14.7 \pm 6.0 \mathrm{U} / \mathrm{g}, \quad 2.2 \pm 0.6 \mathrm{U} / \mathrm{g}$, and $404 \pm 100$ $\min ^{-1} \times \mathrm{g}^{-1}$. The distribution of LMWA in urinary bladder wall is very similar to that of the enzymes. The concentrations of LMWA, except AA, are highest in urothelium and decrease from urothelium to serosa. The concentrations of $\mathrm{AA}$ and GSH in the urothelium sample were $0.75 \pm 0.11$ and $2.7 \pm 0.7 \mu \mathrm{mol} / \mathrm{g}$. The differences between the concentration of antioxidant in the urothelium sample and in other layers are probably even higher, taking into consideration the preparation of the sample. The appropriate amount of urothelium sample needed for the analysis was obtained by combining the first five $20 \mu \mathrm{m}$ thick sections from mucosal tissue pieces. The average thickness of urothelium layer in the middle dorsal bladder body was $68 \pm 17 \mu \mathrm{m}$. Its structure is folded, so that urothelium samples contained some lamina propria. Due to contamination of the urothelium with lamina propria, which contains lower concentrations of antioxidants, the

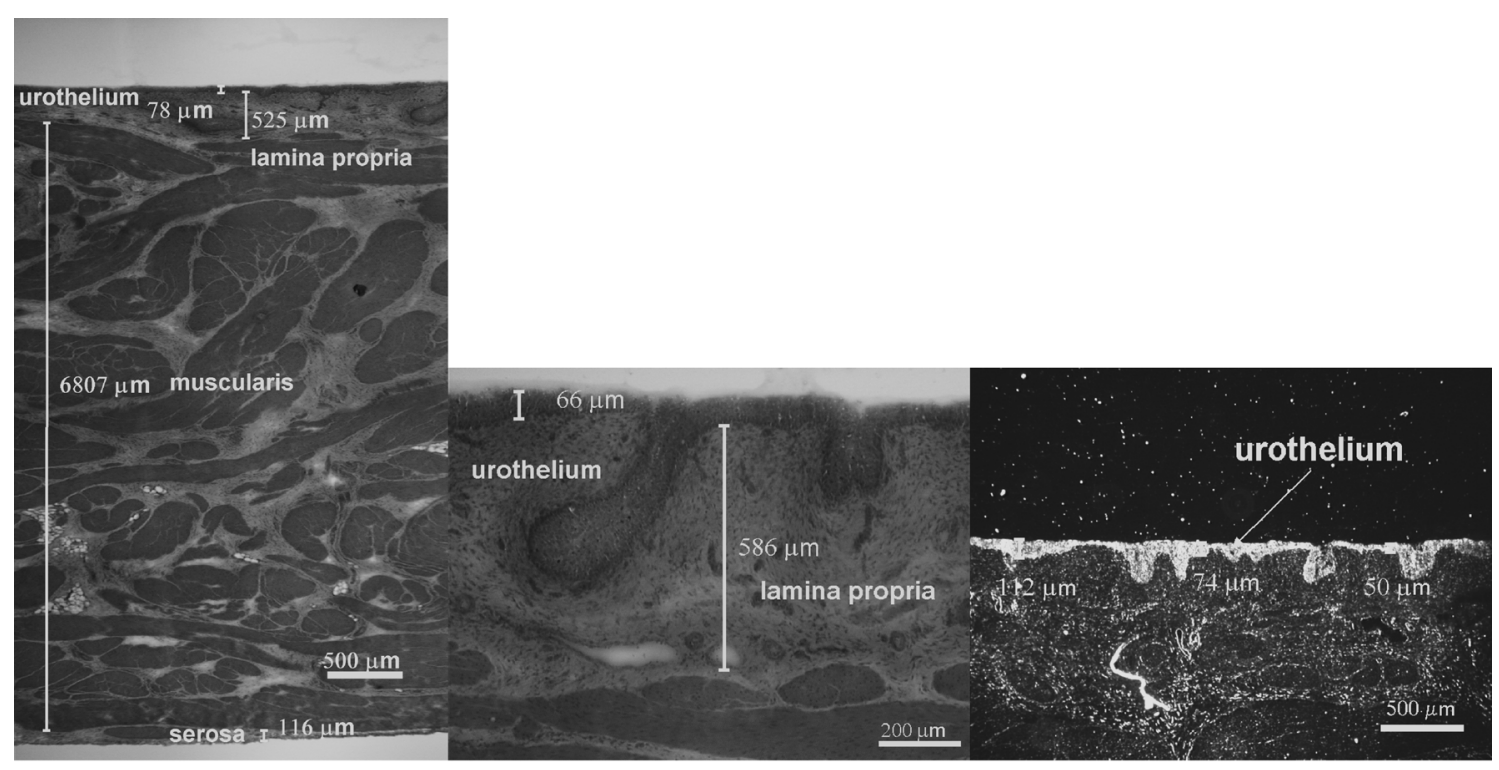

Fig. 2. Histology Figures of Pig Urinary Bladder

Figure presents the urinary bladder wall (A) or urinary bladder mucosa (B, C) obtained after haematoxylin-eosin staining (A, B) or by dark field microscopy (C). The thicknesses of corresponding layers are marked on figures. 


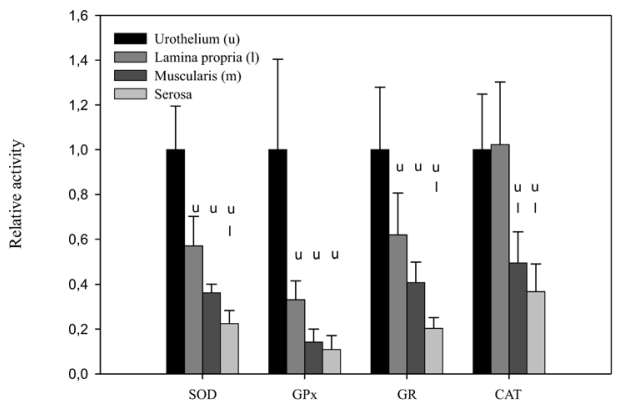

A

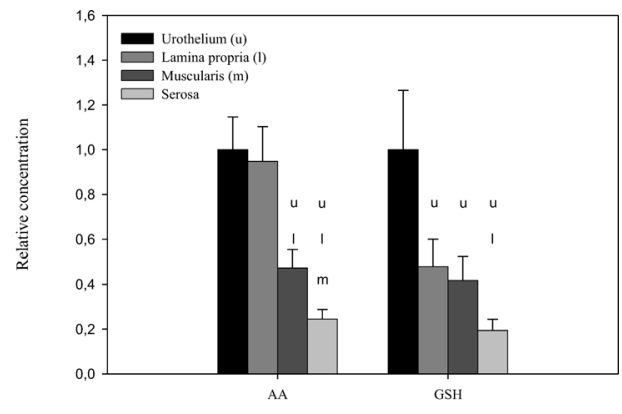

B

Fig. 3. The Activities and Concentrations (Mean \pm S.D.; $n=4-5$ ) of Antioxidant Enzymes (A) and LMWA (B) in the Urinary Bladder Wall Layers

The activities and concentrations are expressed relative to that in urothelium. The symbols indicate significant differences (Bonferroni's test, $p<0.05$ ) between antioxidant activity/concentration in the indicated layer and: urothelium (u), lamina propria (1) and muscularis (m).

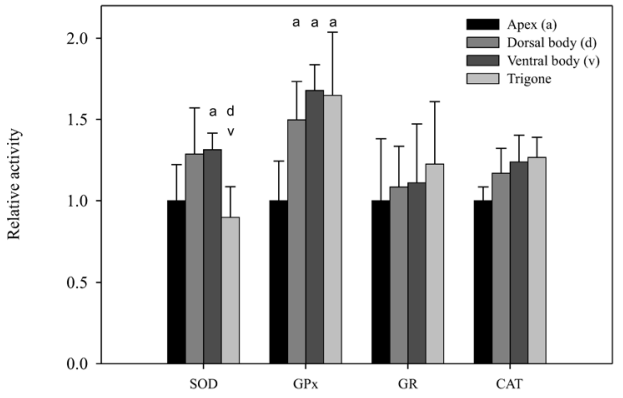

A

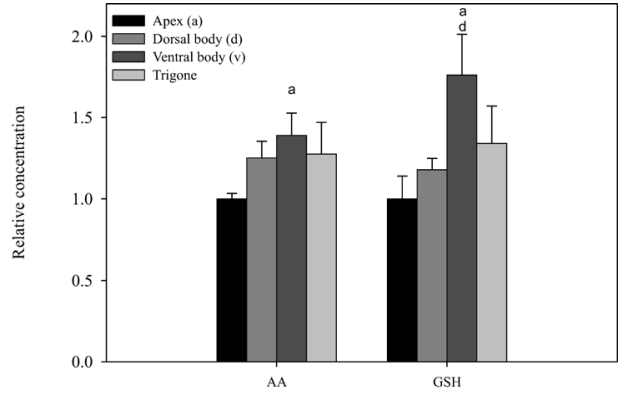

B

Fig. 4. The Activities and Concentrations (Mean \pm S.D.; $n=4-5$ ) of Antioxidant Enzymes (A) and LMWA (B) in the Urothelium Derived from Different Bladder Regions

The activities and concentrations are expressed relative to those in apex. The symbols indicate significant differences (Bonferroni's test, $p<0.05$ ) between antioxidant activity/ concentration in the indicated urothelium region and urothelium derived from: apex (a), dorsal middle bladder body (d), and ventral middle bladder body (v).

measured antioxidant concentrations are probably lower than in the urothelium tissue itself. Nevertheless, all the results clearly demonstrate that antioxidant concentrations are highest in the urothelium.

In the second experiment antioxidant levels in urothelium derived from trigone, middle ventral and dorsal bladder body, and apex were studied on five samples obtained from 20 to 25 pigs (Fig. 4). The activities of SOD, GPx, GR and CAT in the apex urothelium were $433 \pm 96 \mathrm{U} / \mathrm{g}, 10.2 \pm 2.5 \mathrm{U} / \mathrm{g}$, $1.8 \pm 0.7 \mathrm{U} / \mathrm{g}$ and $375 \pm 31 \mathrm{~min}^{-1} \times \mathrm{g}^{-1}$. The concentrations of $\mathrm{AA}$ and GSH in the apex urothelium were $0.61 \pm 0.02$ and $2.1 \pm 0.3 \mu \mathrm{mol} / \mathrm{g}$. ANOVA revealed significant correlations between urothelium regions and SOD, GPx, AA and GSH activity/concentration; the $p$ value for CAT indicated marginal significance. Nevertheless, the results for CAT and GR point towards a distribution similar to that for the other antioxidants studied. The activity of GPx was lower in the urothelium derived from the bladder apex than in all other regions. In the case of SOD, AA and GSH, activities in apex were significantly lower than in urothelium derived from middle ventral bladder body. The results presented in Fig. 4 indicate that the activity/concentration of each studied antioxidant (with the exception of SOD) is lowest in the apex region. Thus, the urothelium apex has lower antioxidant levels than all other regions studied.

\section{DISCUSSION}

The urinary bladder is a specific organ when considering the development of oxidative stress. Increasing amounts of reactive species can be produced in the urine or within the bladder. The mucosa of urinary bladder is constantly exposed to urine, which can contain toxins. Some of the toxins are reactive species, e.g., hydrogen peroxide, others can form reactive species after being metabolized in urinary bladder mucosa or via reactions in the urine. ${ }^{25)}$ As a consequence, mucosal antioxidants can become depleted. Diminished levels of antioxidants can also arise from deficiencies in dietary minerals (e.g., zinc, magnesium, cupper, selenium) or endogenous antioxidants. ${ }^{26}$ Moreover, mutations can affect the activities of antioxidant enzymes. ${ }^{26)}$ Increased production of reactive species is responsible for bladder oxidative stress in the state of ischemia/reperfusion in which depriving oxygen supply (ischemia) followed by re-oxygenation (reperfusion) induces their excessive production. ${ }^{27}$ Finally the excessive activation of endogenous systems producing reactive species, e.g., inducible nitric oxide synthase or myeloperoxidase, can also contribute to the development of oxidative stress in bladder. ${ }^{3,4,8,9,13)}$

Organisms need to adapt to these physiological and pathological conditions. Antioxidants present the first line of defence against oxidative stress. Two of the most important an- 
tioxidant groups are the small group of antioxidant enzymes, including SOD, CAT and GPx, which directly scavenge reactive species, and the second large group of low molecular weight antioxidants (LMWA). Ascorbic acid and glutathione are two of the major water soluble LMWA which react directly with reactive species. GR, GPx and GSH belong to the glutathione system where GR contributes to the maintenance of glutathione in its reduced, antioxidant active form.

The urinary bladder wall consists of four distinct layers: urothelium, lamina propria, muscularis and serosa (urothelium and lamina propria are part of the mucosa). The normal function of urothelium is to provide a non-adherent protective barrier for underlying bladder elements against bacteria, irritating components in the urine, and changes in $\mathrm{pH}$, whereas the detrusor smooth muscle is responsible for mediating micturition through coordinated contraction. The urothelium is directly exposed to the urine and one of its main functions is to prevent urine diffusion into the bladder. As some of urine toxins are reactive species or they can generate reactive species through reactions within the urine or bladder mucosa, we hypothesized that the bladder has to adapt to this stress by concentrating its antioxidants in the urothelium. The distribution of antioxidants in urinary bladder wall clearly confirmed this hypothesis, since antioxidant enzyme activities and LMWA concentrations were the highest in urothelium. The fact that the concentration of antioxidants in urinary bladder wall is progressively lower from urothelium to serosa, suggests that either the majority of oxidative stress derives from the urine or that it is generated within the urothelium or mucosa. Our results are also in accordance with those of Mohandas et al. ${ }^{28)}$ who demonstrated that GSH, GPx and $\gamma$-glutamyl transpeptidase activities were higher in transitional epithelium than in non-transitional rabbit urinary bladder tissue. Additionally, rabbit urinary bladder mucosa exhibits higher SOD and CAT activity than the muscular layer. ${ }^{29,30)}$ Results from our previous study also support the concentration of antioxidants in bladder mucosa. $^{31)}$ These results, however, demonstrate that not only mucosa but rather its thin urothelium layer serves as a defence barrier against reactive species.

Despite the high antioxidant levels in the bladder mucosa, measurement of markers of oxidative damage revealed that oxidative damage is more expressed in mucosa than in the muscular layer. The in vivo rabbit model of partial outlet obstruction, in which ischemia/reperfusion is the basic mechanism of oxidative stress, showed that lipid peroxidation and protein oxidation/nitration were greater in mucosa than in muscular layer. ${ }^{15,27)}$ Since the metabolic rate (phosphate degradation) and blood flow are significantly greater in mucosa than in muscles, the vulnerability of mucosa to ischemia, with consequent oxidative stress, is probably higher. The higher oxidative damage in mucosa could also arise from redox active toxins present in the urine. Toxins can produce oxidative damage also to DNA and together with carcinogens it is possible that they contribute to progression of carcinogenic process. The most common form of urinary bladder cancer is transitional cell carcinoma, which indicates that carcinogenic processes affect mainly urothelium. ${ }^{32)}$

Urothelium is the major defence barrier against reactive species and, in contrast, also the most oxidatively damaged bladder layer. However urothelium is probably not uniformly exposed to oxidative stress and some regional differences can be expected. Our results demonstrate that the urothelium derived from the apex of the urinary bladder has the lowest antioxidant levels of the bladder regions studied. The relative position of the urinary bladder in quadrupeds changes during the normal activities throughout the day. However, most of the time, the apex region is the most inferior part of the bladder. The constant exposure to urine toxins could increase oxidative stress in this region, consequently depleting antioxidants and leading to oxidative damage. Moreover, increased sensitivity to generation of the oxidative stress in the apex is also suggested by the authors who studied the role of neurogenic and mechanical factors in the regulation of blood flow to the bladder base and dome (apex) in the rabbit. ${ }^{33)}$ They found that the neurogenic and mechanical mechanisms appear to favour blood flow to the bladder base more than to the bladder dome, suggesting that the dome may be more vulnerable to ischemia. Low antioxidant levels and increased probability of developing reactive species through an ischemia and reperfusion mechanism, or reactions of toxins in the urine, render the apex region very sensitive to damage. However, regardless of the reason these findings could explain why bladder apex is the most common location of urinary bladder carcinoma in rat. ${ }^{32,34)}$ The carcinogenesis in this region is probably promoted by urinary carcinogens. Moreover increased sensitivity to oxidative damage in this region could potentially contribute to progression of carcinogenesis.

In conclusion, this study indicates that the antioxidants of the urinary bladder are not uniformly distributed in the bladder or urothelial regions. Antioxidants are concentrated in the urothelium and gradually decrease towards the serosa. This implies that urothelium functions as a defence barrier against reactive species. Moreover regional mapping of urothelium demonstrated that among trigone, middle ventral and dorsal bladder body, and apex, the urothelium derived from apex exhibits the lowest antioxidant levels. Based on these findings it can be concluded that apex is the most sensitive to development of oxidative damage and various diseases related to oxidative stress.

\section{REFERENCES}

1) Wolf H., Haeckel C., Roessner A., Virchows Arch., 437, 662-666 (2000).

2) Swana H. S., Smith S. D., Perrotta P. L., Saito N., Wheeler M. A., Weiss R. M., J. Urol., 161, 630-634 (1999).

3) Lemack G. E., Zimmern P. E., Vazquez D., Connell J. D., Lin V. K., J. Urol., 163, 1981-1987 (2000).

4) Austin P. F., Casale A. J., Cain M. P., Rink R. C., Weintraub S. J., J. Urol., 170, 645-648 (2003).

5) Olsson L. E., Wheeler M. A., Sessa W. C., Weiss R. M., J. Pharmacol. Exp. Ther., 284, 1203-1208 (1998).

6) Alfieri A. B., Cubeddu L. X., J. Pharmacol. Exp. Ther., 295, 824-829 (2000).

7) Lundberg J. O., Ehrén I., Jansson O., Adolfsson J., Lundberg J. M., Weitzberg E., Alving K., Wiklund N. P., Urology, 48, 700-702 (1996).

8) Saito M., Wada K., Kamisaki Y., Miyagawa I., Life Sci., 62, PL149PL156 (1998)

9) Saito M., Miyagawa I., J. Urol., 162, 1490-1495 (1999).

10) Durak I., Perk H., Kavutçu M., Canbolat O., Akyol O., Bedük Y., Free Radic. Biol. Med., 16, 825-831 (1994).

11) Yalçin O., Karataş F., Erulaş F. A., Ozdemir E., BJU Int., 93, 863-866 (2004).

12) Malini M. M., Lenin M., Varalakshmi P., Pharmacol. Res., 41, 413- 
418 (2000).

13) Paskaloğlu K., Sener G., Kapucu C., Ayanoğlu-Dülger G., Life Sci., 74, 1093-1104 (2004).

14) Ohnishi N., Liu S. P., Horan P., Levin R. M., Pharmacology, 57, 139147 (1998).

15) Parekh M. H., Lobel R., O’Connor L. J., Leggett R. E., Levin R. M., J. Urol., 166, 341-346 (2001).

16) Aikawa K., Leggett R. E., Levin R. M., J. Urol., 170, 2082-2085 (2003).

17) Levin R. M., Agartan C. A., Leggett R. E., Whitbeck C., Chichester P., Neuman P., Johnson A., Mol. Cell. Biochem., 276, 143-148 (2005).

18) Romanenko A., Morimura K., Wei M., Zaparin W., Vozianov A., Fukushima S., J. Urol., 168, 973-977 (2002).

19) Peskin A. V., Winterbourn C. C., Clin. Chim. Acta, 293, 157-166 (2000).

20) Smith A. D., Levander O. A., Methods Enzymol., 347, 113-121 (2002).

21) Cribb A. E., Leeder J. S., Spielberg S. P., Anal. Biochem., 183, 195196 (1989).

22) Rong Y., Geng Z., Lau B. H. S., Nutr. Res., 16, 1913-1923 (1996).

23) Aebi H., Methods Enzymol., 105, 121-126 (1984).
24) Vovk T., Bogataj M., Roskar R., Kmetec V., Mrhar A., Int. J. Pharm., 291, 161-169 (2005).

25) Halliwell B., Long L. H., Yee T. P., Lim S., Kelly R., Curr. Med. Chem., 11, 1085-1092 (2004).

26) Halliwell B., Whiteman M., Br. J. Pharmacol., 142, 231-255 (2004).

27) Juan Y. S., Lin W. Y., Kalorin C., Kogan B. A., Levin R. M., Mannikarottu A., Urology, 70, 1249-1253 (2007).

28) Mohandas J., Marshall J. J., Duggin G. G., Horvath J. S., Tiller D. J., Cancer Res., 44, 5086-5091 (1984).

29) Onal B., Levin R. M., Kogan B. A., Guven A., Leggett R. E., Mannikarottu A. S., Int. Urol. Nephrol., 39, 1049-1054 (2007).

30) Lin A. D., Mannikarottu A., Kogan B. A., Whitbeck C., Leggett R. E., Levin R. M., Mol. Cell. Biochem., 296, 11-16 (2007).

31) Vovk T., Bogataj M., Mrhar A., Pecar S., Schara M., Biol. Pharm. Bull., 24, 1252-1257 (2001).

32) Frith C. H., Farmer J. H., Greenman D. L., Shaw G. W., J. Environ. Pathol. Toxicol., 3, 103-119 (1980).

33) Kozlowski R., Siroky M. B., Krane R. J., Azadzoi K. M., J. Urol., 168, 1608-1614 (2002).

34) DeSesso J. M., Food Chem. Toxicol., 33, 705-714 (1995). 